Article

\title{
Pratiques et usages de Tinder, une étude exploratoire
}

\author{
Maude Lecompte, Simon Corneau et Dominic Beaulieu-Prévost, \\ Université du Québec à Montréal
}

\section{Résumé}

L'apparition récente des applications de rencontre par géolocalisation, notamment Tinder, est venue modifier les façons de faire des rencontres. Les études actuelles sont peu nombreuses et s'intéressent principalement aux moins de 25 ans. La présente étude vise à documenter les pratiques ainsi que les usages de l'application. Les résultats démontrent que les pratiques sur l'application sont diversifiées, que la séquence d'interactions est préétablie, que les critères de rejet sont nombreux et détaillés, que les qualités de l'application sont à double tranchant et que l'authenticité est un concept polysémique pour les utilisateurs. Les propos recueillis font également ressortir des usages d'intégration, de protection et de performance.

Mots-clés: Tinder, applications, sociologie des usages, nouvelles technologies, rencontres en ligne

\section{Introduction}

La démocratisation de l'accès à Internet et l'amélioration constante des nouvelles technologies de l'information et des communications (NTIC) sont à l'origine d'une multiplication de plateformes numériques destinées aux rencontres sociales, dont les sites pour faciliter les rencontres amoureuses, amicales ou sexuelles, le réseautage professionnel, les réseaux sociaux et plus récemment les applications de rencontre par géolocalisation. Ces applications de rencontre par géolocalisation ont connu un virage important avec l'arrivée des appareils mobiles (téléphones intelligents et tablettes électroniques), qui permettent la recherche de partenaires potentiels partout et en tout temps (Chan, 2017; Ranzini et Lutz, 2017; Tyson, Perta, Haddadi \& Seto, 2016). Parmi les applications disponibles actuellement, Tinder est l'une des plus populaires (Duguay, 2016), avec plus d'un million de jumelages par jour et près de 200 millions 
d'utilisateurs dans plus de 200 pays $^{1}$. L'intérêt populaire pour Tinder est indéniable. À titre d'exemple, le marqueur de métadonnées \#Tinder à lui seul génère plus de 900 000 publications, et ce, seulement sur Instagram ${ }^{2}$. Contrairement aux sites de rencontres traditionnels tels que Réseau Contact, Match.com ou Mon Classeur, Tinder se démarque par son interface conçue pour s'apparenter davantage à celle d'un jeu, soit un jeu de cartes selon ses concepteurs (Ansari \& Klinenberg, 2015; Badili, 2015; Duguay, 2017), et par son fonctionnement simple et ses profils courts (Tyson et al., 2016). Cette simplicité d'usage serait à la base du succès de l'application alors que les abonnés vantent son utilisation rapide et sans effort (David et Cambre, 2016). En matière de fonctionnement, la création d'un profil sur l'application se fait par une connexion au réseau social Facebook ou avec un numéro de téléphone mobile. Les profils contiennent quelques photographies et une description textuelle succincte dont le contenu est à la discrétion des abonnés. L'application permet de circonscrire la recherche en fonction du genre, du groupe d'âge et de la distance. Un profil à la fois est soumis à l'individu qui doit l'évaluer suivant la mention "J'aime » (like ou swipe right), représentée par un cœur ou "Je n'aime pas », dont le symbole est un X (dislike ou swipe left). Lorsque deux personnes se sont mutuellement attribué la mention " J'aime ", il y a un jumelage (match), puis une fenêtre de conversation s'ouvre; elles peuvent dès lors entamer une discussion ou poursuivre leurs recherches.

Malgré la récence d'un intérêt scientifique pour Tinder, les études empiriques actuelles permettent un aperçu des objectifs et des motivations d'utilisation de l'application. Bien que les discours populaires concernant Tinder laissent présager que les objectifs poursuivis par ses abonnés sont principalement de faire des rencontres sexuelles (Ansari \& Klinenberg, 2015), les résultats d'études empiriques n'abondent toutefois pas en ce sens. Dans l'ensemble, les études ont démontré que la recherche de l'amour demeure en tête de liste en termes de motivation d'utilisation (Gatter \& Hodkinson, 2015; LeFebvre, 2017; Hobbs, Owen \& Gerber, 2016; Sumter, Vandenbosch \& Ligtenberg, 2017). D'autres avancent que le divertissement est plus fréquemment mentionné alors que la recherche d'un partenaire arrive ultérieurement dans les préoccupations (James, 2015; Ligtenberg, 2015; Ward, 2016). Plusieurs de ces études comportent certaines limites, notamment des échantillons majoritairement constitués d'adultes émergents (Gatter \& Hodkinson, 2016; Hess, 2014; Hobbs et al., 2016; James, 2015; LeFebvre, 2017; Ligtenberg, 2015; Ranzini, Lutz \& Gouderjaan, 2016; Sevi et al., 2018; Sumter et al., 2017; Timmermans et Courtois, 2018; Timmermans \& De Caluwé, 2018; Tyson et al., 2016), ce qui pourrait expliquer l'occurrence plus élevée d'un usage ludique. En effet, les moins de 30 ans ont accès à

\footnotetext{
${ }^{1}$ Gotinder.com

${ }^{2}$ Service mobile de partage de photographies et de vidéos accessible sur les systèmes d'exploitation IOS, Android et Windows. Instagram appartient au réseau social Facebook.
} 
davantage de contextes de rencontre et à un bassin plus important de partenaires potentiels (Couch \& Liamputtong, 2008; McWilliams \& Barrett, 2014; Stephure, Boon, McKinnon \& Deveau; 2009). En outre, rapporter explicitement le désir de rencontrer un potentiel conjoint comme objectif d'utilisation de Tinder augmenterait avec l'âge (Sumter et al., 2017). Des études se sont quant à elles penchées sur la question des motivations d'utilisation de l'application. Parmi celles-ci, on retrouve la facilité de communication et l'efficacité (Hobbs et al., 2016; Sumter et al., 2017), la validation personnelle (Hobbs et al., 2016; Sumter et al., 2017), l'excitation et l'adhésion aux tendances (LeFebvre, 2017; Sumter et al., 2017). Dans un autre ordre d'idées, Ward (2016) a approfondi les questions de la création, de la modification du profil chez les abonnés, ainsi que de la gestion de son image. Ceux-ci mentionnent éviter de présenter certaines caractéristiques pouvant les associer à des catégories de personnes (ex. image sexualisée associée à une recherche de partenaires sexuels) et modifier leur profil en fonction de la réception des autres tant en termes de mentions like obtenues que de commentaires reçus. Son étude documente également que l'appréciation ou le rejet des profils reposent avant tout sur des caractéristiques liées à l'apparence, mais bifurque rapidement vers des détails précis comme les intérêts et les habitudes de vie inférés par le biais des photographies (Ward, 2016).

Bien que les connaissances actuelles sur Tinder soient embryonnaires, l'augmentation au cours des deux dernières années du nombre de publications scientifiques sur les applications de rencontres, certaines portant plus spécifiquement sur Tinder, démontre l'intérêt grandissant pour cet objet d'étude, tant dans ses modalités d'utilisation qu'à titre de phénomène important du contexte socioculturel actuel. Cet intérêt scientifique pour Tinder outrepasse par ailleurs les frontières disciplinaires alors que les études susmentionnées sont issues de disciplines variées, notamment la psychologie, la sociologie et la communication. L'objectif de la présente étude est d'explorer les pratiques et les usages de Tinder. Outre documenter les pratiques d'utilisation, cette étude vise à répondre à la question suivante: au-delà des façons techniques d'utiliser l'application Tinder et de son utilité annoncée, soit de favoriser des rencontres sociales, quels usages en font ses abonnés? De surcroît, elle comble des lacunes dans les connaissances actuelles sur l'utilisation fonctionnelle de l'application et documente les usages de l'application, et ce, auprès d'un public peu étudié actuellement, soit les adultes d'âge médian. Elle permet aussi de bonifier les connaissances sur l'importance et l'influence des nouvelles technologies dans la vie quotidienne des individus. 


\section{Considérations théoriques}

Cette étude s'inscrit dans le champ de la sociologie des usages, dans la mesure où elle s'intéresse aux pratiques, donc à l'utilisation fonctionnelle de l'application (Jouët, 1993), mais également aux usages. Les usages sont compris comme étant l'aspect symbolique de l'utilisation, la façon d'appréhender l'objet technique et le contexte dans lequel la relation avec l'objet se déploie (Breton \& Proulx, 2006). Dans cette perspective, l'usager n'est pas un récepteur passif de la technologie, il est un joueur actif doté de compétences qui lui permettent de s'approprier les technologies, voire d'en détourner l'utilité basique (Proulx, 2015). Les usagers « bricolent, bidouillent et s'approprient les technologies, et, ce faisant, en viennent à inventer de nouveaux usages non prévus par les concepteurs » (Jauréguiberry, 2015). Cette approche est à propos pour explorer l'utilisation de Tinder, car elle permet non seulement d'appréhender les pratiques, donc les façons de faire effectives sur l'application, mais également, les usages symboliques de l'application et le rapport qu'entretiennent les usagers avec le dispositif.

\section{Méthodologie}

L'échantillon est composé de 33 participants, 16 hommes et 17 femmes. Les critères d'inclusion pour participer à l'étude étaient : 1) être âgé entre 25 et 45 ans, 2) avoir été utilisateur de Tinder au cours de la dernière année, 3) avoir eu au moins une rencontre face à face ou une discussion en ligne avec une personne rencontrée par l'entremise de l'application, 4) être en mesure de s'exprimer en français et 5) résider au Québec. Les participants avaient en moyenne 34 ans ( $m=33,5$, é.t. $=5,7)$, étaient majoritairement domiciliés dans la grande région de Montréal. La durée d'utilisation variait, certains I'utilisant depuis peu alors que d'autres y avaient un profil depuis plusieurs années. Les contextes d'utilisation étaient nombreux tout comme les objectifs poursuivis. Les résultats présentés font état de cette variabilité. La méthode d'échantillonnage a d'abord été un échantillon de volontaires par l'entremise de publications sur divers réseaux sociaux, notamment Facebook, Instagram, LinkedIn $(n=17)$ et sur l'application elle-même $(n=6)$. Ensuite, une méthode boule-de-neige (Beaud, 2009) a permis de compléter l'échantillon ( $n=10)$.

Des entrevues individuelles semi-dirigées ont été réalisées. Cette méthode était pertinente pour l'objet de la recherche, dans la mesure où l'entretien semi-dirigé permet d'approfondir un sujet avec plus de précision, tout en laissant libre-cours aux discours des acteurs à l'intérieur de thèmes présélectionnés (Fenneteau, 2007; SavoieZajc, 2009). Les connaissances actuelles du phénomène ont permis d'identifier des thèmes à approfondir, mais également des thèmes inexploités. Le cadre de l'entrevue 
semi-dirigée permettait ainsi plus de précision et de profondeur dans les contenus abordés, tout en conservant une ouverture à l'expérience individuelle. Le canevas questionnait les éléments suivants: les modalités d'utilisation (comment), les motivations d'utilisation incluant les avantages et les désavantages perçus (pourquoi), les objectifs d'utilisation, les critères de sélection en termes d'appréciation des profils et de raisons de rejet, l'élaboration du profil et sa gestion, le déroulement des interactions et l'opinion générale sur les rencontres en ligne.

Une analyse thématique a d'abord été réalisée sur les transcriptions verbatim des entrevues afin de faire ressortir les grandes tendances ainsi que les particularités de l'expérience d'utilisation de Tinder. Ce type d'analyse vise à « transposer un corpus donné dans un certain nombre de thèmes représentatifs du contenu analysé, et ce, en rapport avec l'orientation de la recherche » (Paillé \& Mucchielli, 2008, p.162). Cette analyse se prêtait bien à notre objet d'étude, car elle permet une vue d'ensemble des pratiques d'utilisation de Tinder et l'émergence progressive d'une compréhension approfondie du phénomène à partir des données recueillies (Deslauriers, 1991; Deslauriers, 1997). Une pré-analyse a permis le développement d'une grille de codification, qui a par la suite servi à l'analyse de toutes les entrevues suivant un processus itératif. L'analyse thématique a été effectuée en deux temps suivant les étapes de décontextualisation et de recontextualisation des données qualitatives (Tesch, 1990). Lors de la première étape (décontextualisation), toutes les entrevues ont été codifiées de façon exhaustive à l'aide du logiciel NVivo 12. Par la suite, l'étape de recontextualisation a permis de regrouper les éléments similaires dans de grandes catégories thématiques, qui rendent comptent de façon succincte des propos recueillis. Les catégories thématiques sont présentées dans la section "Résultats 》. Afin d'assurer la fiabilité des conclusions, des extraits de contenu manifeste témoignent de la congruence entre l'interprétation et le discours des participants. Par ailleurs, le projet de recherche duquel sont issus les résultats a obtenu l'approbation éthique du Comité d'éthique de la recherche pour les projets étudiants de l'Université du Québec à Montréal. Des pseudonymes ont été attribués aux participants dans le but de préserver leur anonymat et les données sociodémographiques sont présentées de façon à éviter toute identification.

\section{Résultats}

\section{Des modalités d'utilisation dynamique}

Les données recueillies font état de pratiques diversifiées sur Tinder tant au niveau des objectifs poursuivis, des contextes physiques et sociaux d'utilisation, que des motivations et de la fréquence d'utilisation. La variété des pratiques ne se décline pas 
seulement en fonction des individus, mais aussi dans l'expérience d'un même individu. Les modalités d'utilisation de Tinder s'inscrivent dans un processus changeant. C'est donc dire que plusieurs facteurs, par exemple la pression des pairs, le succès obtenu, les temps libres et la situation sociale, viennent médier les pratiques.

\subsection{Tinder ce phénomène social : suivre la parade}

Les propos des participants font ressortir que, pour une majorité d'entre eux, le téléchargement initial a été motivé par le désir de suivre la tendance. Certains mentionnant même une utilisation visant à pouvoir échanger avec son réseau social sur un sujet d'actualité, et ce, sans véritable intérêt pour les fonctions annoncées de l'application. L'influence des pairs et le désir de se conformer aux tendances actuelles sont les principaux motifs au téléchargement de l'application. C'est le cas de Daphnée, qui, bien que voulant ultimement faire des rencontres, n'aurait possiblement jamais téléchargé l'application, n'eût été son entourage.

J'entendais souvent parler de cette application par des amis, puis ils rigolaient. C'était drôle, je me suis dit « c'est quoi ce truc ». Ma sœur en fait était dessus alors j'ai demandé à ma sœur, j'ai dit «mais c'est quoi cette application enfin? Est-ce que tu es dessus toi parce que tous mes amis sont dessus [...] ». Après je me suis rendu compte en parlant de ça autour de moi que ma meilleure amie aussi elle était dessus. Donc là je me suis dit « bon, je vais télécharger l'application pour voir ». Mais c'était vraiment par pure curiosité parce que j'avais envie de comprendre pourquoi les gens en parlaient quand ils en parlaient entre eux. $(F, 26)$

C'est dans le rapport à l'autre que la découverte, l'intérêt et la motivation de poursuivre l'exploration des potentialités de l'application se développeraient.

\subsection{Une utilisation qui suit un processus itératif}

L'utilisation de l'application semble, pour la plupart des participants, être cyclique, en ce qu'ils l'utilisent activement, voire compulsivement, pendant une période de temps donnée pour ensuite mettre un terme à son utilisation. Les personnes rencontrées invoquent pour la plupart ce qu'ils qualifient " d'utilisation on and off », donc des allers et des retours fréquents sur l'application. Parmi les raisons invoquées pour expliquer cette tendance, on retrouve notamment l'impression de perdre son temps - une impression jaugée à l'aune d'une baisse observée de la productivité générale (au travail ou dans d'autres sphères de leur vie), ou du manque de succès sur l'application, c'està-dire, un nombre décevant de jumelages avec des personnes jugées intéressantes. Les rencontres significatives sont également susceptibles de mettre un frein à l'utilisation 
alors que les ruptures justifient un retour. Pour Jade $(F, 30)$, une rencontre significative a, plus d'une fois, justifié de se retirer de l'application et des ruptures ont occasionné un retour sur l'application.

La deuxième date que j'ai eue, c'était un gars vraiment intéressant puis j'étais comme « wow il est donc bien génial ». Puis je me suis enlevée, pour justement vivre ce que j'avais à vivre. Moi je n'étais pas à l'aise de vivre deux choses en même temps [...]. Et je l'ai enlevée puis je l'ai remise. Je me suis remise dessus, j'ai eu d'autres dates, mais tant que ce n'était pas agréable, tant qu'on ne se revoyait pas dans un but que peut-être ça marche, je ne m'enlevais pas.

Pour David, c'est l'investissement nécessaire pour rencontrer qui a eu raison de son utilisation. II invoque même la fatigue occasionnée par la demande.

Donc, je rencontrais en série. Je pouvais voir 4-5 personnes par semaine. J'ai fait ça fou raide pendant 3 semaines - 1 mois. Puis là, je n'étais plus capable de fournir, j'étais trop fatigué. $(H, 45)$

\subsection{Jouer à Tinder}

Parmi les principaux objectifs poursuivis, les participants évoquent pour la plupart le divertissement en premier lieu. Tinder permet de «tuer le temps » (Benoit, H, 35) ou, comme le mentionne Thomas $(H, 43)$, de servir de "petit passe-temps ». C'est également le cas en contexte de dyade ou de groupe où l'application fait office de divertissement.

Des fois on va comme swiper en groupe, puis ça devient quelque chose de ludique puis de drôle. (Pascal, H, 30)

Ça animait aussi nos soirées de filles. Moi ça ne me dérangeait pas de le prêter à mes amies pour qu'elles fassent des choix. On riait avec ça. (Jade, F, 30)

\subsection{Le manuel implicite de l'utilisateur}

Malgré la variabilité des contextes, des motivations, des objectifs et des fréquences d'utilisation, des règles implicites semblent régir les pratiques sur l'application. Et ces règles, au surplus, apparaissent comme appuyées par une majorité de personnes interrogées. Elles dictent que l'utilisation de Tinder devrait d'être exploratoire, détachée et sans attente précise, si l'utilisateur souhaite retirer le maximum des avenues offertes par l'application.

Tu écoutes un peu les situations des autres puis tant que tu ne veux rien de sérieux et tu n'as pas d'attentes, vas-y. Mais du moment que tu commences à 
en avoir, premièrement tu vas être déçu, puis en fait si tu n'es pas conscient que justement les gens ne sont pas nécessairement sérieux sur l'application, c'est un petit peu tough. (Jade, F, 30)

En contrepartie, il faudrait éviter la compulsion et la recherche de l'amour à tout prix. Étienne fait état de la bonne façon de procéder pour retirer le plus de bénéfices possible de l'application:

Parce qu'on dirait que les gens qui cherchent l'amour a priori, ils sont toujours déçus puis subissent déception après déception. Même si ça [une rencontre] aboutit dans un lit, ils retournent chez eux le lendemain, déçus, parce qu'ils savent qu'il n'y aura pas de suite. Tandis que quand tu y vas à l'inverse, tu n'es jamais déçu et tu peux juste être agréablement surpris s'il y a une suite. ( $H$, 30)

Les recommandations d'utilisation semblent donc pour la plupart avoir pour objectif d'éviter les déceptions potentielles.

\section{Du premier contact à la rupture du lien : une séquence d'interactions prévisibles}

Nonobstant le fonctionnement basique déterminé par la structure de l'application, une fois un jumelage obtenu, les utilisateurs peuvent ou non poursuivre l'interaction, et ce, de la façon qui leur convient. Malgré cette liberté apparente, la séquence des interactions et les façons de faire sont ressorties comme étant relativement prédéterminées par des conventions.

\subsection{Le premier contact : proactivité et originalité}

En ce qui a trait au premier contact, il est impératif d'être proactif et de se démarquer vu le nombre considérable de personnes sur l'application, voire de conversations simultanées dans lesquelles un usager peut être engagé.

Quand le match se fait, il faut que la conversation aille rapidement sinon bien les matchs se font supprimer assez vite. Donc, si tu travailles pendant la journée et tu n'as pas le temps de répondre, bien ça peut arriver qu'un match du matin, il est parti le soir. (Josée, F, 35)

Considérant que le gars peut, pendant qu'il te parle, parler à 5 autres personnes, et considérant que la fille, pendant qu'elle te parle, peut parler à 5 autres personnes, il faut que tu captes son intérêt assez pour développer une conversation qui a assez de sens pour amener à un « hey ce serait le fun qu'on se rencontre ». (Rachel, F, 31) 
Dans cette optique, les salutations usuelles sont perçues pour la plupart comme sans intérêt, banales et manquant d'originalité, tel que le mentionne Thomas $(H, 43)$ : "C'est basic. Ça part bien trop banal 'Allo, ça va?' ».

\subsection{La rencontre face à face, une question d'efficience}

À la suite des échanges en ligne, les utilisateurs peuvent, à leur guise, poursuivre dans des rencontres en face à face. Les raisons invoquées pour proposer une rencontre, ou différer le moment du premier rendez-vous, relèvent pour la plupart de l'efficience, soit le rapport entre les ressources investies et les résultats obtenus. Les gens veulent savoir rapidement si la personne répond véritablement à leurs attentes, rentabiliser leurs efforts en ligne le cas échéant ou investir dans la rencontre de quelqu'un d'autre, et ce, dans des délais jugés raisonnables. "Ce serait un peu du gaspillage de ne pas se voir », affirme Pascal $(\mathrm{H}, 30)$, pour illustrer

la nécessité de rentabiliser le temps et les efforts investis dans l'interaction.

\subsection{Rompre ou disparaître selon le niveau de matérialisation}

Lorsque vient le temps de mettre fin à l'interaction, l'application permet de supprimer le jumelage. Le profil de l'autre personne n'est alors plus visible et les échanges textuels deviennent impossibles. Le degré d'investissement auprès de l'autre personne ainsi que l'actualisation d'une rencontre en présentiel vont être déterminants dans le choix de la manière la plus adéquate de mettre fin aux échanges. François, par exemple, mentionne qu'il est à l'aise de disparaître sans en aviser son interlocutrice, si I'interaction est demeurée en ligne.

Tandis que si j'ai juste parlé avec la personne sur Tinder en message, bien je n'en ai pas eu de relation humaine avec cette personne-là. Ça ne me dérange pas d'être plus bête. $(H, 27)$

Pour une interaction exclusivement en ligne, la majorité des utilisateurs considèrent le ghosting ${ }^{3}$ comme une option valable. Plusieurs raisons sont mises de l'avant pour justifier cette pratique. Pour David, c'est simplement la façon de procéder sur l'application, compte tenu des fonctions offertes.

Bien là, dans l'application tu peux peser sur le piton « Retirer ». Y a-t-il une raison, aucune raison en particulier, puis pouf ça part. C'est pas mal ça que je fais. [...] Dans ma perception, $C^{\prime}$ est la pratique dans ce réseau social-là. $(H, 45)$

\footnotetext{
${ }^{3}$ Cesser toute communication de façon soudaine et sans avertissement.
} 
Julien $(H, 34)$ pour sa part affirme que «parce que tu es caché derrière une application, il n'y a pas de conséquences ».

3. Des critères de rejet variables et contradictoires : vouloir se ressembler tout en étant différents

Les utilisateurs rapportent que les traits de personnalité, le charisme, les qualités intellectuelles, les intérêts et même certaines caractéristiques physiques peuvent ne pas transparaître dans les profils sur Tinder. Leurs critères de sélection pour tenter d'établir un jumelage relèvent davantage de critères de rejet, en plus d'être contradictoires et variables selon le type de relations recherchées.

\subsection{Rejeter plutôt que choisir}

Alors que le guide d'entretien interrogeait les participants sur les critères de sélection pour un partenaire, une majorité de personnes interrogées disent n'avoir aucun critère de sélection précis, ou être ouvertes à une diversité de personnes. Si elles affirment avoir peu ou pas de préférence, elles invoquent cependant des motifs multiples et précis pour rejeter un profil. Certains vont rejeter sur la base des intérêts et des habitudes de vie; c'est le cas d'Audrey et Mélanie.

\footnotetext{
II ne faut pas qu'il consomme de la drogue. II ne faut pas que sa religion lui dicte son quotidien. Il faut qu'il boive, mais avec modération parce que moi je ne bois pas. II ne faut pas qu'il fume, oh mon dieu. Ça, c'est très concret comme critère, mais ça en élimine vraiment beaucoup malheureusement. II faut qu'il soit ambitieux de moyen à élevé, mettons. (Audrey, F, 31)

Moi quelqu'un qui a un verre dans les mains, ça, c'est non, moi je ne consomme pas. Quand ta photo de profil tu as un verre dans les mains, ça dit aussi que dans la vie de tous les jours, tu aimes prendre un verre. Moi je ne me match pas avec quelqu'un qui va prendre un verre tous les jours. (Mélanie, F, 42)
}

Alors que d'autres rejettent davantage en fonction de la personnalité et de I'attitude comme Jean :

II ne faut pas que la personne soit, je ne veux pas dire bitch, mais high maintenance. Moi le monde qui sont fermés, généralement ce sont du monde qui ne sont pas trusting. (Jean, $\mathrm{H}, 40$ )

Certains vont même discriminer des candidats en fonction de leur lieu de résidence. 
La région géographique je dirais, de plus en plus elle a un impact. Je ne sortirai jamais avec une fille de Laval. (Maxime, $\mathrm{H}, 36$ )

Les participants rencontrés verbalisent plus aisément les éléments qui les poussent à rejeter des candidats que les caractéristiques recherchées. D'ailleurs, Jean $(H, 40)$ mentionne que le fonctionnement de Tinder amène à analyser les profils en vue de les filtrer sur la base d'éléments qui sont moins appréciés. Il estime procéder à une forme d'élimination.

\subsection{L'importance du dimorphisme sexuel}

Tant chez les hommes que chez les femmes, le dimorphisme sexuel semble une caractéristique prisée. Le dimorphisme sexuel concerne les différences morphologiques entre les hommes et les femmes (Larsen, 2003; Lewis, 2011). Pour la totalité des personnes interrogées, la personne qui endosse le rôle le plus féminin doit être de plus petite taille, même dans un contexte non hétérosexuel.

Plus grand que moi, mais il n'a pas besoin de mesurer 6 pieds. [...]

Probablement 5 pieds 7 en montant. (Cynthia, F, 32)

Moi ça c'est vraiment important, une fille qui a une plus petite shape que la mienne. Je ne sais pas si c'est parce que d'habitude moi je suis plus masculine et j'aime ça sentir que je suis comme un peu protectrice. (Jacynthe, F, 26)

Tant chez les hommes que chez les femmes, ce critère est suffisamment déterminant pour encourager l'inscription de sa taille à même son profil. Les participants, précisément, y voient une manière d'augmenter leurs chances d'obtenir des jumelages réussis ou d'éviter les déceptions.

Je mets ma grandeur, parce que, oui ça je le mets au début, parce qu'il y a des filles qui sont grandes, elles ne veulent pas des gars petits. C'est ça. Je le mets. [...] Juste comme début, après cela je vais mettre un petit texte. Là, elles voient ça que je suis grand parce qu'il y en a qui cherchent des gars grands. (Marc, $H, 43)$

Bien que cette caractéristique soit davantage de l'ordre du physique, la recherche de la différence ne semble pas transposable aux caractéristiques sociales recherchées chez des partenaires potentiels comme en témoignent les résultats qui suivent. 


\subsection{La recherche de l'homogamie}

Parmi les critères de sélection les plus récurrents dans l'appréciation d'un profil et le choix de poursuivre l'interaction après le jumelage, on retrouve l'homogamie de diplôme et de classe sociale. L'homogamie est la concordance des caractéristiques sociales de deux individus dans une optique de relation sociale et/ou affective (Bozon \& Heran, 2006). Par exemple, Julien, Jacynthe et Marc souhaitent rencontrer quelqu'un qui ait un statut social et professionnel semblable au leur.

J'ai des études supérieures et tout, je vais accorder quand même de l'importance à la scolarité. Je ne serais pas trop du type à être avec une coiffeuse. (Julien, H, 34)

Pour moi aussi les études c'est très important. Pas que je ne serais pas avec quelqu'un qui n'a pas un baccalauréat, c'est juste que moi, si tu me dis que tu travailles à l'épicerie du coin et que tu n'as pas l'intention de sortir de là puis essayer de faire autre chose... (Jacynthe, F, 26)

La profession, j'avoue depuis qu'ils ont ajouté ça, ça m'aide. [...]. Je pense qu'on se comprend mieux des fois dans des professions. (Marc, $\mathrm{H}, 43$ )

\section{L'application qui a les défauts de ses qualités}

Les utilisateurs interrogés font état de plusieurs points positifs à l'utilisation de Tinder. Par contre, ces mêmes qualités semblent apporter leur lot de défauts. Pour plusieurs, ces défauts sont suffisamment nombreux pour justifier une opinion somme toute négative.

\subsection{Facile, accessible et flexible, mais fonctionnalités limitatives}

Parmi les principales qualités de l'application, la simplicité de la plateforme et la création instantanée du profil semblent satisfaire les individus rencontrés.

Ça se fait tellement rapidement et facilement que ce n'est pas tellement compliqué. (Maxime, $\mathrm{H}, 36$ )

Parce que c'est facile d'accès, pas besoin de t'écrire un compte à n'en plus finir. Tu mets 3-4 photos. (François, H, 27)

Les utilisateurs soulignent aussi la flexibilité temporelle et géographique offerte par l'application au chapitre des options intéressantes. Tinder leur offre la possibilité de faire des rencontres dans le confort de leur domicile ou au travail, à l'heure de leur 
choix, ainsi que de chercher des partenaires dans un périmètre rapproché, ou au contraire, d'étendre leurs recherches à un plus large territoire.

Tinder c'est un bar qui ne ferme jamais où il y a une immensité de personnes continuellement. C'est comme un buffet chinois toujours ouvert que tu peux aller littéralement $24 \mathrm{~h}$ sur 24 , où tu peux parler à quelqu'un $24 \mathrm{~h}$ sur 24. (Rachel, F, 31)

Je trouve que Tinder ça m'apporte une porte à un monde social qui fait que je peux rencontrer des gens dans le confort de mon salon et discuter. (Josée, $F, 35)$

En contrepartie, ils estiment que plusieurs des fonctions de l'application nuisent parfois au processus de rencontre. C'est le cas notamment de la nécessité d'un jumelage pour aborder un autre utilisateur.

\subsection{Beaucoup d'options, beaucoup de gestion}

Les utilisateurs vantent l'étendue des possibilités offertes par Tinder en termes de quantité d'utilisateurs, de diversité de profils et d'accès à de nouveaux réseaux de contacts.

Des fois tu croises des gens dans tes réseaux sociaux, au travail ou dans des bières, c'est tout le temps les mêmes personnes. Tu te dis « ah wow il y a tout un monde à découvrir ». Moi je l’ai téléchargée à cause de ça. Voir si je ne tombais pas sur des genres de gars sur qui je n'étais jamais tombée avant. (Coralie, F, 28)

Par contre, ce même potentiel est à l'origine de désagréments quant à la gestion de ses contacts sur l'application. En effet, la multiplicité des options est perçue comme nécessitant un investissement important pour faire des choix et rencontrer les candidats potentiels.

Là tu te ramasses que tu textes avec plein de monde en même temps. II faut comme que tu choisisses aussi. II y a comme un enjeu de qu'est-ce que je priorise pour rencontrer. Parce que là un moment donné c'est bien beau rencontrer du monde. Ça vient « demandant » entre guillemets. (Catherine, $F, 30$ )

\subsection{L'interface: protection ou obstruction}

Les utilisateurs voient pour la plupart l'interface comme un atout, en ce sens que I'application diminue la gêne et l'appréhension du rejet, mais également comme un 
désagrément, dans la mesure où le non verbal échappe aux interactions. À ce propos, Gaël et Élyse font état de la plus-value des rencontres en ligne dans la prise de contact, mais également pour éviter le rejet.

\footnotetext{
Moi je trouve que ça facilite la vie. Tu n'as pas besoin de sortir, rencontrer du monde. Moi ça me gêne trop de toute façon. S'il n'y avait pas ça, je pense que je serais célibataire pour le restant de mes jours. (Gaël, H, 36)

Si tu démontres de l'intérêt pour quelqu'un qui n'en a pas pour toi, bien la personne ne le saura pas, alors tu évites le rejet. (Élyse, F, 25)
}

D’un autre côté, Benoit et Julie abordent quant à eux les limites imposées par l'interface, le fait notamment qu'elle nuise au contact humain et limite la capacité à apprécier d'autres éléments que l'apparence.

Définitivement, il y a une barrière informatique qui départage les deux êtres humains et tout ce qui est essentiel à l'être humain, le contact physique, le regard [...]. Oui, il y a cette coupure, ce manque de proximité humaine. (Benoit, $\mathrm{H}, 35$ )

Quand on a un premier contact avec quelqu'un, même si la personne n'est pas nécessairement belle physiquement, il peut y avoir une attirance, il peut y avoir le charme et tout. Ça, on peut difficilement l'avoir avec une application parce qu'on n'a pas ce contact-là. (Julie, F, 28)

\section{Quand authenticité rime avec présenter le meilleur de soi-même}

Parmi les éléments attendus des profils, l'authenticité et la perception de réalisme sont déterminantes. L'authenticité attendue est cependant à modalités variables. Plusieurs mentionnent le naturel comme étant garant de cette authenticité; néanmoins ce naturel se doit d'être crédible aux yeux des autres utilisateurs.

Des profils où est-ce qu'il y a 4 photos, promenade de dromadaire, bungee jumping, escalade pis sur une moto, c'est quoi ta vie? Ce n'est pas toi ça, on dirait que tu es juste un catalogue pour un magasin de plein air. (Rachel, F, 31)

Les utilisateurs recherchent l'équilibre dans les intérêts présentés, ou plutôt inférés, dans les profils. Par exemple, un profil contenant exclusivement des photographies prises lors de voyages sera évalué comme peu représentatif de la réalité par les autres utilisateurs, au même titre que des images trop travaillées laisseront présager un faux profil.

Il faut se vendre un peu c'est normal. Si ça a l'air trop fake c'est câlisse, ça fait vraiment louche. Ça ne $\mathrm{m}^{\prime}$ inspire pas confiance. (Pascal, $\mathrm{H}, 30$ ) 
Les utilisateurs veulent voir des photographies représentatives tant en termes de récence que de naturel.

Je trouve cela un peu hypocrite dans le sens, tu sais, les gens qui vont mettre une photo d'eux, mais depuis cette photo-là tu as pris 35 livres. C'est de vendre du rêve. (François, $\mathrm{H}, 27$ )

En contrepartie, ils s'attendent à des profils originaux, qui se démarquent. Ce à quoi ils portent une attention particulière dans la création de leur propre profil.

Je me rends compte que c'est plus accrocheur les choses comme ça que juste marquer sportif puis le bon vin et les amis. Ça te prend des petites phrases qui se démarquent. (Thomas, $\mathrm{H}, 43$ )

J'évite des phrases du style « j'aime le bon vin et les sushis entre amis ». Ah oui wow c'est original, tu manges, c'est hot. (Luc, H, 36)

\subsection{Penser son profil pour en optimiser les résultats}

Bien que l'authenticité et la ressemblance soient attendues des autres sur l'application, la plupart des utilisateurs font des choix stratégiques dans la création de leur profil pour maximiser leurs chances. Étienne, par exemple, mentionne utiliser des stratégies graphiques pour bonifier son image : « J'aime beaucoup les photos en noir et blanc. Ça cache les défauts ». $(H, 30)$

Nombreux sont les utilisateurs qui ont également apporté des changements à leur profil en cours d'utilisation pour en augmenter la productivité en termes de jumelages. C'est le cas de Jean $(H, 40)$, qui affirme avoir fait des tests pour « voir si quelque chose donnait plus de matchs » ou Martin $(H, 39)$, qui a fait des changements pour que "ça marche plus». Parmi les principales modifications mentionnées, on retrouve notamment l'ajout de photographies plus avantageuses et le retrait d'informations ou d'images susceptibles de diminuer leurs chances de succès sur I'application. À ce chapitre, Étienne $(H, 30)$ admet avoir fait des modifications à son profil pour cacher son tabagisme, tandis que Martine $(F, 39)$ a choisi de retirer les informations sur son occupation principale.

Au début, j'avais écrit de quoi puis ça ne marchait pas, fack je l'ai enlevé. Je disais ce que je faisais puis je disais que j'étais étudiante puis ce n'était pas winner. $(F, 39)$ 


\section{Discussion}

Dans l'ensemble, les concepteurs de l'application semblent avoir atteint leur objectif d'amener les utilisateurs à percevoir l'application comme un jeu (Duguay, 2017). Les pratiques démontrent l'adhésion à cette idée chez les utilisateurs de l'application qui I'utilisent pour se divertir et rire, seul ou en groupe. L'usage ludique de l'application amène d'ailleurs à considérer les autres abonnés comme de simples profils, de là la possibilité d'adopter des comportements qui seraient inacceptables dans d'autres circonstances, dont le ghosting. L'utilisation déclarée de Tinder s'apparente davantage à ce que l'on sait des réseaux sociaux, c'est-à-dire, une utilisation quotidienne, sans objectifs précis et intégrée à une routine, qui diffère des sites de rencontres traditionnels (Mull \& Lee, 2014 ; Ryan, Chester, Reece \& Xenos, 2014; Ligtenberg, 2015). Les objectifs poursuivis dans l'utilisation de Tinder sont similaires à ceux documentés précédemment auprès d'adultes émergents (James, 2015; Ligtenberg, 2015; Ward, 2016), et ce, malgré certaines études ayant fait ressortir que les individus de plus de 30 ans auraient moins de raison d'utiliser les rencontres en ligne sans véritable désir de rencontrer (Brym \& Lenton, 2001; Sumter et al., 2017).

Mis à part les constats factuels liés aux pratiques, les propos recueillis ont fait ressortir trois usages dominants de l'application : l'intégration, la protection ainsi que la performance. Pour reprendre les propos de Jauréguiberrry (2015), l'intégration concerne "la nécessité d'être intégré à un ensemble de réseaux pour exister économiquement et socialement »(p.199). Avoir un profil Tinder permet d'intégrer une communauté d'utilisateurs, de suivre les tendances du moment et d'échanger avec d'autres personnes sur son utilisation comme l'ont mentionné les participants à l'étude. L'ubiquité technologique permet l'intégration dans plusieurs réseaux. Toutefois, le contact avec l'autre occasionne des risques potentiels, notamment le rejet, mais également la remise en question de son identité, un risque qui semble toutefois minimisé sur Tinder. Giddens (1994) mentionnait par ailleurs que l'individu est maintenant délivré des dangers et des craintes situationnelles grâce aux technologies et les propos recueillis font état de cette protection de l'interface. Tinder permet de se protéger contre le rejet, mais également contre la déception en offrant le filtre nécessaire à un premier tri minutieux.

La performance, quant à elle, renvoie à l'utilisation de l'application dans le dessein d'augmenter sa performance générale, d'être rentable, efficace, efficient et compétitif (Jauréguiberry, 2015). Cet usage transparaît notamment dans les avantages perçus de l'application qui sont explicités en termes d'efficacité, de rapidité et de possibilités d'action. Les avenues offertes par Tinder permettent non seulement d'être performant dans son utilisation de l'application, mais également dans les autres 
sphères de la vie. Les désavantages répertoriés tournent également autour de la notion de performance, dans la mesure où certaines critiques concernent des fonctions de I'application qui peuvent retarder ou nuire à l'obtention de plus d'affinités, donc à une plus grande performance. D'ailleurs, lorsque les ressources investies sur l'application sont plus grandes que les résultats obtenus, les personnes rencontrées ont tendance à se désinvestir de l'application, car elle n'est plus rentable. C'est le cas également lorsque l'usage de Tinder nuit à la performance dans d'autres sphères la vie. L'importance de la performance transparaît également dans la séquence d'interactions, dont la nécessité de se démarquer, mais également, d'éviter toute perte de temps. L'efficience recherchée dans la rencontre en face à face s'inscrit dans ce désir de performance. Tinder permet donc, outre ses fonctions explicites de rencontres sociales, d'être intégré à une communauté, de se protéger des risques inhérents à la rencontre d'autrui et d'être socialement performant.

\section{Conclusion}

Cette étude permet non seulement de documenter les pratiques d'utilisation de l'application, mais également son aspect symbolique. Les rencontres en ligne demeurent un sujet hautement polarisé. Alors que certains auteurs célèbrent les avancées technologiques au nom de la libéralisation, de la possibilité de maintenir des liens au-delà de la présence physique et d'une plus grande réflexivité (Kessous, 2011; Zerbib, 2012), d'autres y voient une forme de déshumanisation assistée numériquement, ou une rationalisation de la quête amoureuse caractéristique de la postmodernité (Bergström, 2012; 2013 Illouz, 2006; Lardellier, 2014a, 2014b). On observe d'ailleurs ces deux tendances dans les usages. D'une part, l'usage de Tinder témoigne d'une volonté d'être en lien avec l'autre, notamment par le désir de suivre la tendance, de faire partie du groupe et d'ultimement faire des rencontres. D'autre part, le désir de se protéger et de performer laisse à penser que l'utilisation soit motivée a priori par des considérations individualistes. Au-delà de ces nouvelles connaissances sur les pratiques et les usages de Tinder, les perceptions et les significations attribuées à l'application par ses utilisateurs méritent d'être approfondies dans des recherches ultérieures, afin de mieux comprendre ses effets sur les fondements des aspirations sociales et amoureuses.

\section{Limites}

D’emblée, il importe de relativiser les résultats obtenus en tenant compte des limites liées au corpus. De fait, le corpus est composé principalement de résidents de la grande région de Montréal, aussi peut-on penser que certaines conclusions soient moins représentatives hors des grands centres urbains, par exemple l'utilisation ludique de 
I'application. Pour profiter pleinement de Tinder, encore faut-il qu'il y ait une quantité d'utilisateurs suffisante pour rendre cette utilisation attrayante. On peut penser également que certains des avantages et désavantages de l'application sont modulés par la quantité de profils sur l'application, donc que l'appréciation de Tinder diffère selon la densité de population.

\section{Références}

Ansari, A. et Klinenberg, E. (2015). Modern Romance. Penguin Books.

Badili, B. (2015). The implications of doing gender in the digital world of dating. [Mémoire de maîtrise, Gonzaga University, Spokane, Washington, États-Unis].

Beaud, J-P. (2009). L'échantillonnage. Dans B. Gauthier (dir.), Recherche sociale : de la problématique à la collecte de données (5e édition, p. 251-283). Presses de I’Université du Québec.

Bergström, M. (2012). Nouveaux scénarios et pratiques sexuels chez les jeunes utilisateurs de sites de rencontres. Agora / Débats Jeunesses, Presses de Sciences Po, 60, 107-119.

Bergström, M. (2013). La loi du supermarché? Sites de rencontres et représentations de l'amour. Ethnologie française, 3(43), 433-442.

Bozon, M. et Héran, F. (2006). La formation du couple. La Découverte.

Breton, P. et Proulx, S. (2006). L'explosion de la communication. Introduction aux théories et aux pratiques de la communication. La Découverte.

Chan, L.S. (2017). Who uses dating apps? Exploring the relationships among trust, sensation-seekink, smartphone use, and the intent to use dating apps based on the Integrative Model. Computers in Human Behavior, 72, 246-258.

Couch, D. et Liamputtong, P. (2008). Online dating and mating : the use of the Internet to meet sexual partners. Qualitative Health Research, 18(2), 268-279.

David, G. et Cambre, C. (2016). Screened intimacies : Tinder and the swipe logic. Social Media + Society, 2, 1-11.

Deslauriers, J-P. (1991). Recherche qualitative : Guide Pratique. McGraw-Hill Éditeurs. 
Deslauriers, J.-P. (1997). L'induction analytique. Dans Poupart, J., Deslauriers, J-P., Groulx, L-H., Laperrière, A., Mayer, R. et Pires, A.P. (Éds), La recherche qualitative : Enjeux épistémologiques et méthodologiques (p. 293-308). Gaëtan Morin.

Duguay, S. (2017). Dressing up Tinderella : interrogating authenticity claims on the mobile dating app Tinder. Information, Communication \& Society, 20(3), 351367.

Fenneteau, H. (2007). Enquête : entretien et questionnaire (2e éd). Dunod.

Gatter, K. et K. Hodkinson. (2015). On the differences between Tinder versus online dating agencies: Questioning a myth. An exploratory study. Cogent psychology, 3, 1-12.

Hess, Rachel, E. (2014). Love in the time of smartphones: a comparative analysis of the dating application "Tinder"”. [Mémoire de maîtrise. University of South Alabama, Mobile, Alabama, États-Unis].

Giddens, A. (1984). The constitution of society: outline of a theory of structuration. University of California press.

Hobbs, M. Owen, S. et Gerber, L. (2016). Liquid Love? Dating apps, sex, relationships and the digital transformation of intimacy. Journal of Sociology, 53(2), 271-284.

Illouz, E. (2006). Réseaux amoureux et Internet. Réseaux, 4(138), 269-272.

James, J. L. (2010). Mobile dating in the digital age: computer-mediated communication and relationship building on Tinder. [Mémoire de maîtrise, Texas State University, San Marcos, Texas, États-Unis].

Jauréguiberry, F. (2015). Les technologies de communication : d'une sociologie des usages à celle de l'expérience hypermoderne. Cahiers de recherche sociologique, (59-60), 195-209.

Jauréguiberry, F. (2003). Les branchés du portable, Sociologie des usages. Presses universitaires de France. 
Kessous, E. (2011). L'amour en projet : Internet et les conventions de la rencontre amoureuse. Réseaux, 2(166), 191-223.

Lardellier, P. (2014a). De la monogamie au « polygaming »... : le « papillonnage » numériquement assisté, nouveau paradigme sentimentalo-sexuel. Sociologie et sociétés, 46 (1), 103-124.

Lardellier, P. (2014b). Le libéralisme à la conquête de l'amour. Quelques constats et réflexions sur la consommation sentimentale et sexuelle de masse à l'ère d'Internet. Revista româna de sociologie, 24(3-4), 163-176.

Larsen Spencer, C. (2003). Equality for the sexes in human evolution? Early hominid sexual dimorphism and implications for mating systems and social behavior. Proceedings of the National Academy of Sciences of the United States of America, 100(16), 9103-9104.

LeFebvre, L. (2017). Swiping off my feet : Explicating relationship initiation on Tinder. Journal of Social and Personal Relationships, 35(9), 1205-1229.

Lewis, S.K. (2011). Gendering the body: exploring the construction of the sexually dimorphic body. [Mémoire de maîtrise. Portland State University, Portland, Oregon, États-Unis].

Ligtenberg, Loes. (2015). Tinder, the app that is setting the date scene on fire: a uses and gratifications perspective. [Mémoire de maîtrise, Graduate School of Communication, Amsterdam, Pays-Bas].

McWilliams, S. et Barrett, A.E. (2012). Online Dating in Middle and Later Life : Gendered Expectations and Experiences. Journal of Family Issues, 35(3), 411436.

Mull, I.R. et Lee, S.E. (2014) «PIN » : pointing the motivational dimensions behind Pinterest. Computers in Human Behavior, 33, 192-200.

Proulx, S. (2015). La sociologie des usages, et après? Revue française des sciences de l'information et de la communication, 6, 1-13.

Ryan, T., Chester, A., Reece, J. et Xenos, S. (2014). The uses and abuses of Facebook: A review of facebook addiction. Journal of Behavioral Addictions, 3(3), 1-16. 
Paillé, P. et Mucchielli, A. (2008). L'analyse qualitative en sciences humaines et sociales. Armand Colin.

Ranzini, G., Lutz, C. et Gouderjaan, M. (2016, juin). Swipe Right : An exploration of selfpresentation and impression management on Tinder. Communication présentée à la 66th Annual Conference of the International Communication Association (ICA), Fukuoka, Japon.

Ranzini, G. et Lutz, C. (2017). Love at first swipe? Explaining Tinder self-presentation and motives. Mobile Media \& Communication. 5(1), 80-101.

Savoie-Zajc, L. (2009). L'entrevue semi-dirigée. Dans B. Gauthier (dir.), Recherche sociale: de la problématique à la collecte de données (5e édition, p. 337-360). Presses de l'Université du Québec.

Sumter, S.R., Vandenbosch, L. et Ligtenberg, L. (2017). Love me Tinder : Untangling emerging adults' motivations for using the dating application Tinder. Telematics and Informatics, 34, 67-78.

Sevi, B., Aral, T. et Eskenazi, T. (2018). Exploring the hook-up app : Low sexual disgust and high sociosexuality predict motivation to use Tinder for casual sex. Personality and Individual Differences, 133, 17-20.

Stephure, R.J., Boon, S.D., MacKinnon, S.L. et Deveau, V.L. (2009). Internet initiated relationships: Associations between age and involvement in online dating. Journal of Computer-Mediated Communication, 14, 658-681.

Tesch, R. (1990). Qualitative Research : Analysis types \& software tools. Routledge.

Timmermans, E. et Courtois. C. (2018). From swiping to casual sex and/or committed relationships: Exploring the experiences of Tinder users. The Information Society, 34(2), 59-70.

Timmermans, E. et De Caluwé, E. (2017). To Tinder or not to Tinder, that's the question: An individual differences perspective to Tinder use and motives. Personality and Individual Differences, 110, 74-79.

Tyson, G., Perta, V.C., Haddadi, H. et Seto, M.C. (2016). A first look at User Activity on Tinder. Communication présentée à la International Conference on Advances in Social Networks Analysis and Mining (ASONAM), San Francisco, États-Unis. 
Ward, J. (2016). What are you doing on Tinder? Impression management on a matchmaking mobile app. Information, Communication \& Society, 20(11), 1644-1659.

Zerbib, O. (2012). Écris-moi et tu te diras qui tu es : Les sites de rencontre comme lieux de réenchantement de soi. Le temps des médias, 2(19), 66-86. 